\title{
Investigation of the Kerr effect in a blue phase liquid crystal using a wedge-cell technique
}

\author{
Kamil Orzechowski, ${ }^{* 1}$ Marek W. Sierakowski, ${ }^{1}$ Marzena Sala-Tefelska, ${ }^{1}$ Tomasz R. Woliński, ${ }^{1}$ \\ Olga Strzeżysz, ${ }^{2}$ and Przemysław Kula ${ }^{2}$ \\ ${ }^{1}$ Faculty of Physics, Warsaw University of Technology, Koszykowa 75, 00-662 Warsaw, Poland \\ ${ }^{2}$ Institute of Chemistry, Military University of Technology, 00-908 Warsaw, Poland
}

Received May 29, 2017; accepted June 28, 2017; published June 30, 2017

\begin{abstract}
In this work an alternative method has been described for refractive index measurement of a blue phase liquid crystal in the Kerr effect. The proposed wedge method uses a simple goniometric setup, allowing for direct index measurements for any wavelengths and index values. This is a significant advantage comparing to other methods, usually having limitations of the measurement range as well as requiring complicated calculation to obtain refractive index values. The results are reliable and agree well with subject literature.
\end{abstract}

Great interest in blue phase liquid crystals (BPLCs) for their prospective applications in future photonic technology still persists. BPLC is a highly chiral material, formed as a self-assembling nanostructure with threedimensional periodicity in the spatial arrangement of defects [1-3]. To date, three structurally distinct blue phases have been found: BP III, BP II, and BP I, appearing in this order of decreasing temperature from the isotropic to chiral phase and existing in comparatively narrow temperature ranges, typically $0.5-2 \mathrm{~K}$. The BPs possess unique electro-optic properties that make them attractive for photonic and display applications. In particular, they exhibit selective Bragg reflections, optical isotropy and polarization insensitivity in a macroscopic scale, ultra-fast switching speed that is faster than in nematics by an order of magnitude, and reveal birefringence induced by an external electric field (Kerrlike effect). Any prospective design of photonic devices utilizing a BPLC requires determining their refractive index (indices) of material under external conditions, i.e. temperature or electric field [4-7]. Consequently, a convenient and accurate measurement method of the BPLC refractive index (indices), in particular in the electro-optical Kerr effect is an important issue in material characterization.

At present, many methods offer high accuracy measurements of liquid crystal refracting indices. However, refractive index measurements in the Kerr effect of the BPLC are more complicated than in ordinary nematics. In a typical planar BPLC-sample (that assumes homogeneous distribution of an electric field) the optical

*E-mail: orzechowski@if.pw.edu.pl axis of an initially isotropic cell is induced along the direction of light propagation. Therefore, the changes of a refractive index in the perpendicular direction to the optical axis, affecting the light transmitted through the sample, are relatively small. In literature, a few measurement methods such as interferometric or the Senarmont method were reported [8-10]. All the methods require complicated calculations to obtain refractive index values. In this letter, we present an alternative, and simpler wedge method for refractive index measurement in the bulk BP sample. The proposed wedge method uses a less complicated experimental setup described by us elsewhere [11], which is based on measuring the position of the light refracted by a wedge-shaped cell filled with the investigated BPLC. The setup is schematically shown in Fig. 1. To obtain higher accuracy of our measurements, a CCD camera was used to determine the beam positions.

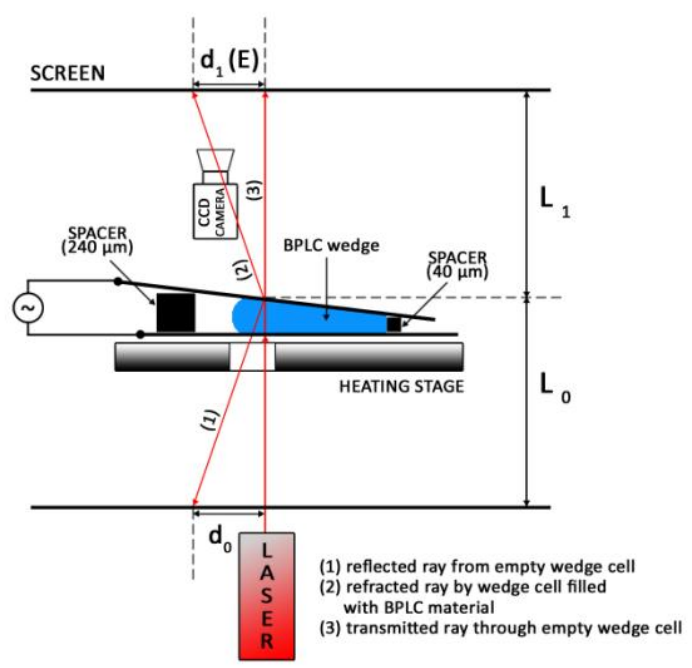

Fig. 1. Experimental setup to measure a refractive index of BPLC in the Kerr effect. 


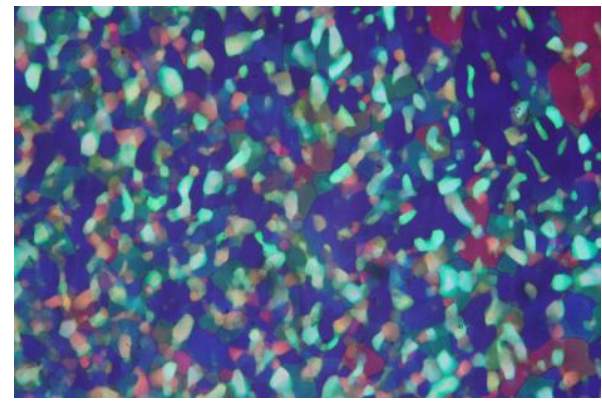

Fig. 2 Polycrystalline structure of BPLC observed in the wedge-shaped cell by using polarized optical microscopy.

The BPLC used in our experiment was a mixture consisting of nematics 1912 ( $86 \%$ by wt.) of the components described elsewhere [12] and two optically active chiral dopants: biphenyl-4,4'-dicarboxylic acid bis(1-methyl-heptyl) ester and [1,1';4',1']terphenyl-4,4"'dicarboxylic acid bis-(1-methylheptyl) ester (both $7 \%$ by wt.). All the chemical substances were synthesized at the Military University of Technology. The wedge cell was made of two glass plates with an ITO coating to enable measurements under the influence of an external electric field. Since the wedge angle is small (about 2-3 degrees) we can assume an almost homogeneous distribution of the electric field inside the BPLC sample.

The structure of the BPLC in the wedge-shape sample during measurements was typically polycrystalline as shown in Fig. 2.

In a voltage-off state, the BPLC can be treated as an optically isotropic material with the average refractive index [13]:

$$
n_{\text {avg }}=\frac{2 n_{o}(E)+n_{e}(E)}{3},
$$

where: $n_{o}(E)$ and $n_{e}(E)$ are the field-dependent refractive indices: perpendicular (ordinary) and parallel (extraordinary) to the electric field, respectively.

The electric field applied to the sample induces a change in the refractive index $\delta n(E)$ of the BPLC. The index change along the beam direction (optic axis) is given by:

$$
\delta n_{\|}(E) \equiv n_{e}(E)-n_{\text {avg }}=\frac{2\left(n_{e}(E)-n_{o}(E)\right)}{3},
$$

which does not affect the transmitted light. However, the index change $\delta n_{\|}$causes also modification of the refractive index in the direction perpendicular to the beam via the electrostriction effect, which is expressed by the following relation:

$$
\delta n_{\perp}(E) \equiv n_{\text {avg }}-n_{o}(E)=\frac{n_{e}(E)-n_{o}(E)}{3} .
$$

As a result, an induced birefringence $\Delta n_{\text {ind }}(E)=n_{e}(E)-$ $n_{o}(E)$ appears. The change of $\delta \mathrm{n}_{\perp}$ shifts the refracted beam position $\left(d_{1}\right)$, which can be directly measured in the setup shown in Fig. 1.

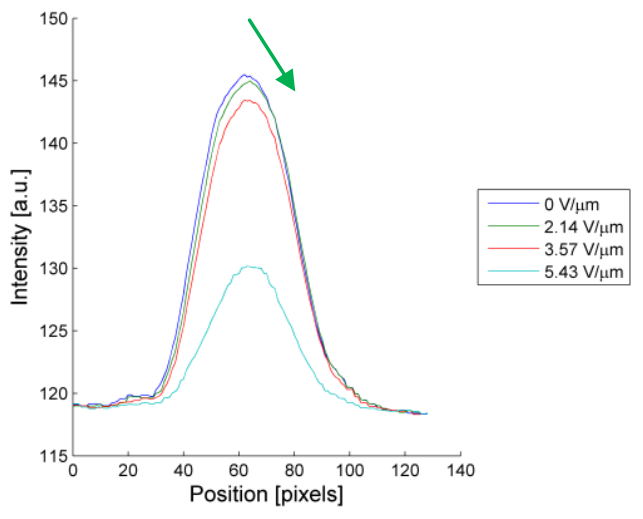

Fig. 3 Profile and position of a refracted beam for different intensities of the electric field.

The position of a refracted beam as a function of the electric field is presented in Fig. 3. A small shift of the refracted beam for various intensities of the electric field is visible on the graph. Note an asymmetry of the shift appearing as a larger displacement of the left edges of the curves. Moreover, for a sufficiently high electric field (at $5.43 \mathrm{~V} / \mu \mathrm{m})$, a dramatic decrease in beam intensity occurrs. This is due to the fact that the focal conic texture of the chiral phase is induced locally in the bulk sample causing elevated light scattering [14]. From the shifts measured in our setup we can obtain the values of $n_{o}(E)$ according to the following relation [11]:

$$
n_{o}(E)=\left(1+\frac{d_{1}(E)}{L_{1}} \cdot \frac{2 L_{0}}{d_{0}}\right),
$$

where: $d_{0}$ is the distance between spot reflected from the empty wedge cell and the setup axis, $d_{l}(E)$ is the distance between the axis and refracted spot for the cell filled with BPLC, and $L_{0}, L_{l}$ are the distances between the source of light to the sample and from the sample to the screen, respectively.

In this way we can obtain also $n_{\text {avg }}$ for $\mathbf{E}=0$. If the average and ordinary refractive indices ( $n_{\text {avg }}$ and $n_{o}$, respectively) were obtained from the experimental data measured directly, then the extraordinary refractive index $\left(n_{e}\right)$ was calculated from Eq. (3).

The experimental data of refractive indices in the Kerr effect of BPLC are presented in Fig. 4. The measurements were done at $58.0^{\circ} \mathrm{C}$. Theoretical fitting curves for the experimental data were obtained by using the extended Kerr model in the following forms [13]:

$$
\begin{aligned}
& n_{o}(E)=n_{\text {avg }}-\frac{1}{3} \Delta n_{\text {sat }}\left(1-\exp \left(-\left(E / E_{\text {sat }}\right)^{2}\right)\right), \\
& n_{e}(E)=n_{\text {avg }}+\frac{2}{3} \Delta n_{\text {sat }}\left(1-\exp \left(-\left(E / E_{\text {sat }}\right)^{2}\right)\right),
\end{aligned}
$$


where: $\Delta n_{\text {sat }}$ and $E_{\text {sat }}$ correspond to the saturated values of the induced birefringence and electric field, respectively.

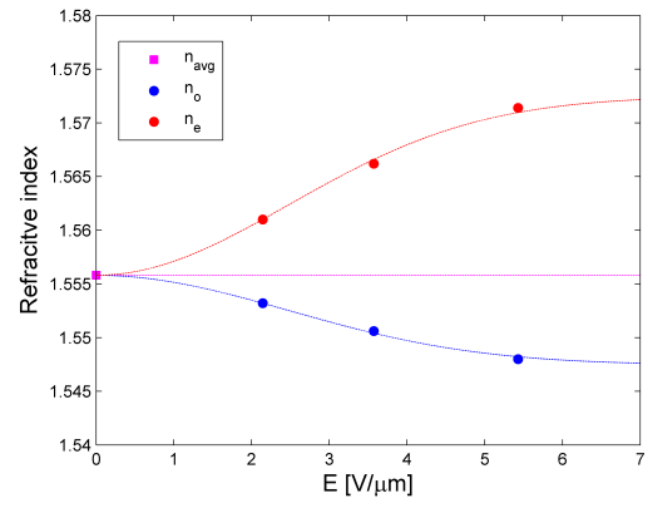

Fig. 4. Refractive indices of BPLC as a function of an electric field measured for a laser diode $(\lambda=640 \mathrm{~nm})$. The fitting curves (red and blue)

were obtained from Eqs. (5a) and (5b). The straight magenta line

corresponds to the average refractive index of the investigated BPLC.

The values of refractive indices of the BPLC for red laser light are relatively high. Still higher values can be expected for shorter wavelengths due to chromatic dispersion of the LC material. It is worth mentioning that our method allows for index measurement of any wavelengths and index values, which is a significant advantage in comparison with other methods, usually having limitations of the measurement range.

The induced birefringence $\Delta \mathrm{n}_{\text {ind }}$ of the BPLC is shown in Fig. 5. It is clearly seen that the Kerr effect for the BPLC can be approximated by the classical Kerr formula with $\Delta n=\lambda K E^{2}$, where $\mathrm{K}$ is the Kerr constant [15], only in the low-electric field region. However, the induced birefringence data agree well with the extended Kerr model proposed by J. Yan et al. [13]. By fitting the extended Kerr model to our data, the Kerr constant was determined as $3.19 \mathrm{~nm} / \mathrm{V}^{2}$ (for $\Delta n_{\text {sat }}=0.025, E_{\text {sat }}=3.5 \mathrm{~V} / \mu \mathrm{m}$, $\lambda=0.64 \mu \mathrm{m})$.

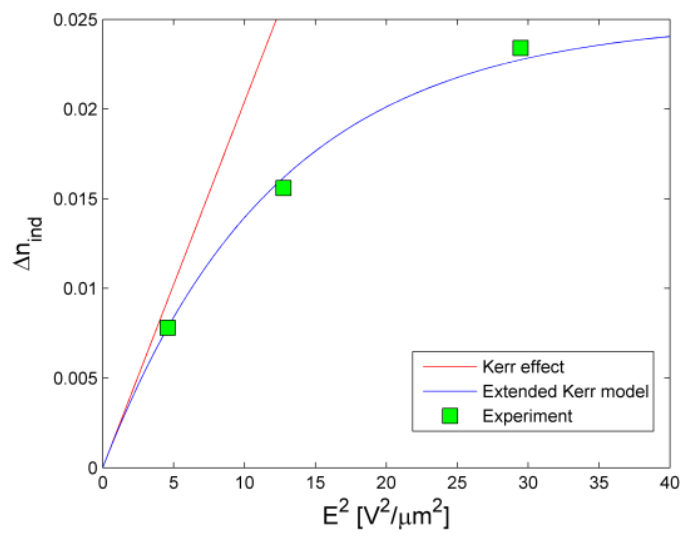

Fig. 5. Electric field dependent birefringence of BPLC (green squares). The fitting curves correspond to the classic Kerr effect (red straight line) and extended Kerr model (blue line).
The value of the Kerr constant for the measured material is close to but a little higher than $2.1 \mathrm{~nm} / \mathrm{V}^{2}$ given in the work [12], obtained by another method. The observed difference may come from the stabilization technique of the BPLC. In Ref. [12], the authors used polymerstabilization of BPLC, which resulted in higher electric fields required to reorient BPLC molecules and in the decreased Kerr constant.

To conclude, an alternative method for refractive index measurement in the Kerr effect has been investigated. This simple goniometric method gives results in good agreement with literature. Among important advantages of the proposed wedge method are the lack of limitations for measured refractive index values, i.e. the possibility to measure high refractive index, and no need to use either polarized or coherent beams.

The authors would like to acknowledge the grant from National Science Centre, Poland 2015/19/D/ST3/02432.

\section{References}

[1] W. Cao et al., Nat. Mater. 1, 111 (2002).

[2] S. Meiboom, M. Sammon, W.F. Brinkman, Phys. Rev. A. 27, 438 (1983).

[3] S. Tanaka et al., Sci. Rep. 5, 16180 (2015).

[4] Y. Li, S.-T. Wu, Opt. Express 19(9), 8045 (2011).

[5] N. Rong et al., J. Disp. Technol. 12(10), 1008 (2016).

[6] J.-D. Lin et al., Opt. Express 22(24), 29479 (2014).

[7] P. Joshi et al., Photon. Lett. Poland 9(1), 11 (2017).

[8] Ch.-W. Chen et al., Opt. Mater. Express 3(5), 527 (2013).

[9] Y.-H. Lin et al., Appl. Phys. Lett. 109, 104503 (2011).

[10] J. Yan et al., Opt. Express 18(11), 11450 (2010).

[11] K.A. Rutkowska, K. Orzechowski, M. Sierakowski, Photon. Lett. Poland 8(2), 51 (2016).

[12] O. Chojnowska et al., Appl. Phys. Lett. 116, 213505 (2014).

[13] J. Yan et al., Appl. Phys. Lett. 96, 071105 (2010).

[14] M. Chen et al., Opt. Mater. Express 4(5), 953 (2014).

[15] J. Kerr, Philos. Mag. 50, 337 (1875). 